\title{
NON-CLASSICAL INTEGRALS OF BESSEL FUNCTIONS
}

\author{
S. N. STUART
}

(Received 14 August 1979)

(Revised 20 June 1980)

\begin{abstract}
Certain definite integrals involving spherical Bessel functions are treated by relating them to Fourier integrals of the point multipoles of potential theory. The main result (apparently new) concerns

$$
\int_{0}^{\infty} d t t^{\left|t_{1}-l_{2}\right|+2 N+2} j_{l_{1}}\left(r_{1} t\right) j_{1_{2}}\left(r_{2} t\right)
$$

where $l_{1}, l_{2}$ and $N$ are non-negative integers, and $r_{1}$ and $r_{2}$ are real; it is interpreted as a generalized function derived by differential operations from the delta function $\delta\left(r_{1}-r_{2}\right)$. An ancillary theorem is presented which expresses the gradient $\nabla^{2 n} Y_{l m}(\nabla)$ of a spherical harmonic function $g(r) Y_{L M}(\Omega)$ in a form that separates angular and radial variables. A simple means of translating such a function is also derived.
\end{abstract}

\section{Introduction}

Some of the best-known differential equations of mathematical physics lead to Fourier integrals that involve Bessel functions when, for instance, they are solved under conditions of rotational symmetry about a centre. As long as the integrals are absolutely convergent they are amenable to the classical analysis of Watson's standard treatise, but a calculus of wider serviceability can be expected from the theory of generalized functions. This paper identifies a class of Bessel-function integrals that are Fourier integrals of derivatives of the Dirac delta function in three dimensions. They arise in the practical context of manipulating special functions-notably when changing the otigin of the polar coordinates, for example, in order to evaluate so-called two-centre integrals and 
convolutions [6, 11 (equation 22), 12, 14, 17]. A simple translation formula is presented which serves to indicate that context.

The translation formula would find application in calculating the potential integrals, overlap integrals, molecular integrals, cluster integrals, collision integrals and so on which variously describe the classical, quantum and statistical mechanics of atomic interaction.

An underlying theme of this work is my belief that calculations of the above type are facilitated by an interplay of direct and Fourier methods.

\section{Single Bessel function}

The infinite integral,

$$
\int_{0}^{\infty} d t t^{\mu} J_{v}(r t)
$$

has been defined classically by first damping the integrand and then resorting to the limit of no damping. Thus [18, page 514, and 21, page 391]

$$
\lim _{p \rightarrow 0+} \int_{0}^{\infty} d t t^{\mu} J_{\nu}(r t) e^{-p t}=\frac{\Gamma\left(\frac{1}{2}+\frac{1}{2} \nu+\frac{1}{2} \mu\right)}{\Gamma\left(\frac{1}{2}+\frac{1}{2} \nu-\frac{1}{2} \mu\right)} \times \frac{2^{\mu}}{r^{\mu+1}} .
$$

If one defines $\nu_{n} \equiv \nu+2 n+1$, where $n$ is a non-negative integer, then it will be seen that, if $0<\mu \neq \nu_{n}$, the above expression diverges as $r \rightarrow 0$. On the other hand, if $r \neq 0$, the expression vanishes as $\mu \rightarrow \nu_{n}>n$, when the lower gamma function becomes singular. There is clearly a deep singularity at the point $r=0$, $\mu=\nu_{n}>n$. It is here argued that this integral and others like it can be interpreted usefully as generalized functions.

We shall be restricting the discussion to half-odd-integer values of the parameters, which means spherical Bessel functions,

$$
j_{l}(2 \pi u) \equiv \frac{1}{2} u^{-1 / 2} J_{I+1 / 2}(2 \pi u),
$$

and we shall rely upon their connexion [19, page 410 and 21, page 50],

$$
i^{l} j_{l}(2 \pi r k) Y_{l m}\left(\Omega_{\mathrm{r}}\right)=(4 \pi)^{-1} \int d \Omega_{\mathrm{k}} e^{2 \pi r r . k} Y_{l m}\left(\Omega_{\mathrm{k}}\right),
$$

with the spherical harmonics $Y_{l m}(\Omega)$ in $R^{3}$, the integer $l$ being non-negative and $\Omega$ denoting spherical polar coordinates $(\theta, \phi)$ [2] pages 18-19 and [9] pages 494-495. This approach will allow us to harness the rotation-group theory and algebra of spherical harmonics which, as Sack has well observed, are practically indispensable nowadays [15]. 
Let us begin with the Dirac delta function in $R^{3}$, whose Fourier representation is

$$
\delta(\mathbf{r})=\int d^{3} \mathbf{k} e^{2 \pi i r \cdot \mathbf{k}},
$$

where the integral is understood as a generalized function [7] pages 197-198, and repeated differentiation of which gives a tensor,

$$
\nabla^{2 n+l} \delta(\mathbf{r})=\int d^{3} \mathbf{k} e^{2 \pi i r . k}(2 \pi i \mathbf{k})^{2 n+l}
$$

We now define the differential operator $Y_{l m}(\nabla)$ as a component of an irreducible spherical tensor, directly analogous to the regular Laplace harmonic $Y_{l m}(\mathbf{r}) \equiv$ $r^{l} Y_{l m}\left(\Omega_{\mathrm{r}}\right)[1],[4]$ page 127 , and [16] appendix 2 . It should be noted that $Y_{l m}(\nabla)$ is proportional to the irreducible spherical-tensor component $(\mathrm{lm})$ of the differential monomial $\nabla^{\prime}$. Then we can at once derive the Fourier transform,

$$
\nabla^{2 n} Y_{l m}(\nabla) \delta(\mathbf{r})=(2 \pi i)^{2 n+l} \int d^{3} \mathbf{k} e^{2 \pi i r \cdot k} k^{2 n} Y_{l m}(\mathbf{k})
$$

This derivative of the delta function may be regarded as representing the point multipole $(\mathrm{n} / \mathrm{m})$ of potential theory [2] pages 72-74 and [13]. Making an angular integration according to equation (1), we obtain

$$
\int d^{3} \mathbf{k} e^{2 \pi r \cdot k_{k}} k^{2 n} Y_{l m}(\mathbf{k})=4 \pi i^{l} Y_{l m}\left(\Omega_{\mathbf{r}}\right) \int_{0}^{\infty} d k \cdot k^{2 n+l+2} j_{l}(2 \pi r k)
$$

By acquiring a Bessel function in this way the radial integral assumes the form considered previously, with $\nu=l+\frac{1}{2}$.

Direct factorization is another means of separating the angular and radial variables of the point multipole, for which the following theorem is helpful.

TheOREM 1. (Chain rule). If $f$ is a generalized function of position in $R^{3}$ and depends only on the radius $r$, then

$$
Y_{l m}(\nabla) f(r)=Y_{l m}(\mathbf{r})\left(r^{-1} \partial / \partial r\right)^{l} f(r)
$$

in the sense of generalized functions, namely

$$
\int d^{3} \mathbf{r} \gamma(\mathbf{r}) Y_{l m}(\nabla) f(r)=\int d^{3} \mathbf{r} f(r)\left(r^{-1} \partial / \partial r\right)^{\dagger l} Y_{l m}(\mathbf{r}) \gamma(\mathbf{r})
$$

where the adjoint (denoted by a dagger) is $\left(r^{-1} \partial / \partial r\right)^{\dagger \prime}=(-1)^{l} r^{-1}\left(r^{-1} \partial / \partial r\right)^{l} r$.

Proof. This starts from the Wigner Eckart theorem, which allows the case of $m=l$ to suffice [2] pages 56-59 and [3] pages 77-79, and from the fact that $Y_{l l}(\nabla)$ is proportional to the monomial $(\partial / \partial x+i \partial / \partial y)^{l}$; the rest of the argument is similar to Jones' theorem on $\nabla^{2} f(r)$ [7] pages 215-216. (Incidentally the result (4) applies also to functions in the ordinary sense [4] page 175.) In 
addition there are the following lemmas on radial differentiation of the delta function,

$$
\begin{aligned}
\nabla^{2 n} \delta(\mathbf{r}) & =(-2)^{n} n !\left(r^{-1} \partial / \partial r\right)^{n} \delta(\mathbf{r}) \\
& =\delta^{(2 n+2)}(r) / 4 \pi(n+1),
\end{aligned}
$$

which can be proved by means of adjoints using an arbitrary test-function $\gamma \sim r^{2 N}$, and further [7] pages 62-63,

$$
r^{l} \delta^{(k)}(r) / k !=(-1)^{l} \delta^{(k-l)}(r) /(k-l) ! \quad(k>l) .
$$

From equations (4)-(7) it follows that

$$
Y_{l m}(\nabla) \nabla^{2 n} \delta(\mathbf{r})=\frac{2^{n} n !(2 n+2 l+1) ! !}{2 \pi(2 n+l+2) !} Y_{l m}\left(\Omega_{r}\right) \delta^{(2 n+l+2)}(r)
$$

so that the variables are separated.

Assembling equations (2), (3) and (8), and equating their radial parts, we have the result,

$$
\int_{0}^{\infty} d k(2 \pi k)^{2 n+l+2} j_{l}(2 \pi r k)=(-1)^{n+1} 2^{n-1} n ! \frac{(2 n+2 l+1) ! !}{(2 n+l+2) !} \delta^{(2 n+l+2)}(r),
$$

which represents the integral as a generalized function localized at $r=0$. The same formula has been obtained, rather more directly, by Kay, Todd and Silverstone [8] equation (24).

\section{Two Bessel functions}

While in the previous section an integral over one Bessel function has been derived from a point multipole, it will now be shown that an integral over two Bessel functions can be derived in a similar way by translating the point multipole. It may be recalled that generalized functions can be translated by the following Fourier transform technique [12], [14] and [17]:

$$
\left.\begin{array}{rl}
f\left(\mathbf{r}_{1}+\mathbf{r}_{2}\right) & =\int d^{3} \mathbf{k} e^{2 \pi i \mathbf{r}_{1} \cdot \mathbf{k}} e^{2 \pi i \mathbf{r}_{2} \cdot k \bar{f}(\mathbf{k})} \\
\bar{f}(\mathbf{k}) & =\int d^{3} \mathbf{r} e^{-2 \pi i \mathbf{k} \cdot \mathbf{r}} f(\mathbf{r}) .
\end{array}\right\}
$$

It is convenient in what follows to treat angular variables by the methods of Racah algebra, and to define the coupled basis [3] page 36 with elements,

$$
\left\langle\Omega_{1} \Omega_{2} \mid l_{1} l_{2} ; L M\right\rangle=\sum_{m_{1} m_{2}}\left\langle\Omega_{1} \mid l_{1} m_{1}\right\rangle\left\langle\Omega_{2} \mid l_{2} m_{2}\right\rangle\left\langle l_{1} m_{1}, l_{2} m_{2} \mid L M\right\rangle
$$


where $\langle\Omega \mid l m\rangle$ is just the spherical harmonic $Y_{l m}(\Omega)$ in Dirac notation, and the final factor is a Clebsch Gordan coefficient. The tensors (11) have been called bipolar harmonics [2] pages 55-56. Their virtue is that they present the same sort of closure relation (a Laplace series) as do the spherical harmonics of which they are composed [4] pages $342-359$ and [9] pages 494-495:

$$
\begin{gathered}
\sum_{l m}\langle\Omega|| m\rangle \int\left\langle\operatorname{lm} \mid \Omega^{\prime}\right\rangle d \Omega^{\prime}\left\langle\Omega^{\prime} \mid f\right\rangle=\sum_{l m}\langle\Omega \mid l m\rangle\langle\operatorname{lm} \mid f\rangle=\langle\Omega \mid f\rangle, \\
\sum_{l_{1} l_{2} L M}\left\langle\Omega_{1} \Omega_{2} \mid l_{1} l_{2} ; L M\right\rangle\left\langle l_{1} l_{2} ; L M \mid f\right\rangle=\left\langle\Omega_{1} \Omega_{2} \mid f\right\rangle .
\end{gathered}
$$

By this means a function of two positions can be expanded in terms of bipolar harmonics. In the case of $f\left(r_{1}+r_{2}\right)$, the expansion contains the following scalar product,

$$
\begin{aligned}
\left\langle l_{1} l_{2} ; L M \mid f\left(\mathbf{r}_{1}+\mathbf{r}_{2}\right)\right\rangle= & (4 \pi)^{2}\left\{\frac{\left(2 l_{1}+1\right)\left(2 l_{2}+1\right)}{4 \pi(2 L+1)}\right\}^{1 / 2}\left\langle l_{1} 0, l_{2} 0 \mid L 0\right\rangle i^{l_{1}+l_{2}} \\
& \times \int d^{3} \mathbf{k} j_{l_{1}}\left(2 \pi r_{1} k\right) j_{l_{2}}\left(2 \pi r_{2} k\right)\left\langle L M \mid \Omega_{\mathbf{k}}\right\rangle \bar{f}(\mathbf{k}) .
\end{aligned}
$$

Here we have used equations (11), (10), and then (1) for the angular integration over $\Omega_{1}$ and $\Omega_{2}$, together with the fundamental composition theorem of spherical harmonics [2] page 57 and [3] page 164,

$$
\left\langle l_{1} l_{2} ; L M \mid \Omega \Omega\right\rangle=\left\{\frac{\left(2 l_{1}+1\right)\left(2 l_{2}+1\right)}{4 \pi(2 L+1)}\right\}^{1 / 2}\left\langle l_{1} 0, l_{2} 0 \mid L 0\right\rangle\langle L M \mid \Omega\rangle .
$$

When the generalized function to be translated is the point multipole,

$$
f(\mathbf{r})=\nabla^{2 n} Y_{l m}(\nabla) \delta(\mathbf{r}),
$$

the requisite Fourier transform is provided by equation (2):

$$
\bar{f}(\mathbf{k})=(2 \pi i)^{2 n+l} k^{2 n} Y_{l m}(\mathbf{k}) \text {. }
$$

On substitution of this into equation (14), and after angular integration,

$$
\begin{aligned}
&\left\langle l_{1} l_{2} ; L M \mid \nabla_{1}^{2 n} Y_{l m}\left(\nabla_{1}\right) \delta\left(\mathbf{r}_{1}+\mathbf{r}_{2}\right)\right\rangle \\
&=\langle L M \mid l m\rangle 4\left\{\frac{\left(2 l_{1}+1\right)\left(2 l_{2}+1\right)}{4 \pi(2 l+1)}\right\}^{1 / 2}\left\langle l_{1} 0, l_{2} 0 \mid l 0\right\rangle i^{l_{1}+l_{2}+l+2 n} \\
& \times \int d k(2 \pi k)^{2 n+l+2} j_{l_{1}}\left(2 \pi r_{1} k\right) j_{l_{2}}\left(2 \pi r_{2} k\right)
\end{aligned}
$$

The matrix element vanishes unless $L=l,\left(l_{1}+l_{2}+l\right)$ is even, and the usual triangular condition is mutually satisfied:

$$
\left|l_{1}-l_{2}\right|<l<l_{1}+l_{2}
$$


The positive power of $k$ in the integrand of equation (16) means that we have a Weber Schafheitlin integral which classically is not convergent [21] page 399. The classical expressions assuming convergence vanish, both for the case of $r_{1} \neq r_{2}$ and at the discontinuity $r_{1}=r_{2}$, because of a singular gamma function occurring as a denominator [21] equations 13.4(2) and 13.41(2).

Direct angular integration is another means of separating the angular and radial variables of the translated point multipole. To that end we invoke the following gradient theorem.

THEOREM 2. If $g$ is a generalized function of position in $R^{3}$ and depends only on the radius $r$, then

$$
\left\langle l_{1} m_{1}\left|\nabla^{2 n} Y_{l m}(\nabla) g(r)\right| l_{2} m_{2}\right\rangle=\left\langle l_{1} m_{1}\left|Y_{l m}(\Omega)\right| l_{2} m_{2}\right\rangle D_{n l l_{1} l_{2}}(r) g(r),
$$

in the sense of generalized functions, namely

$$
\begin{aligned}
\int d^{3} \mathbf{r} \gamma(r)\left\langle l_{1} m_{1}\left|\nabla^{2 n} Y_{l m}(\nabla) g(r)\right| l_{2} m_{2}\right\rangle & \\
= & \left\langle l_{1} m_{1}\left|Y_{l m}(\Omega)\right| l_{2} m_{2}\right\rangle \int d^{3} \mathbf{r} g(r) D_{n l l l_{1}}^{\dagger}(r) \gamma(r) .
\end{aligned}
$$

Here $D$ denotes the radial differential operators,

$$
\begin{aligned}
D_{n l_{1} l_{2}}(r) & \equiv(-1)^{n+b} D_{n+a, l_{1}} D_{n+b, l_{2}}^{\dagger} \\
& =(-1)^{l_{2}} D_{l_{1} l_{1}} D_{10}^{2(n-c)} D_{l_{2} l_{2}}^{\dagger} \quad(n>c),
\end{aligned}
$$

and

$$
D_{N J} \equiv r^{J}\left(r^{-1} \partial / \partial r\right)^{N} r^{N-J}
$$

where

$$
2 a \equiv l+l_{1}-l_{2}, \quad 2 b \equiv l+l_{2}-l_{1}, \quad 2 c \equiv l_{1}+l_{2}-l .
$$

Proof. As it was for Theorem 1 of the previous section, proof is by way of the Wigner Eckart theorem. This treatment is more general (and possibly less cumbrous in form) than the results of Santos and Bayman [1] and [16] appendix 2, which are restricted to the case of $n=0$. The case of $n=l_{2}=b=c=0$, $l_{1}=l=a$, reduces to the previous theorem.

The power of the theorem may be indicated by applying it to Bessel functions. If one takes equation (1) as a definition of the Bessel function, then equation (17) can be used to generate a differential relation between two such functions,

$$
(-1)^{n+a} D_{n l_{1} l_{2}}(r) j_{l_{2}}(r t)=t^{2 n+l_{j_{1}}}(r t) \text {. }
$$

This formula contains Bessel's equation as a special case, $n=1, l=a=b=0$, $l_{1}=l_{2}=c$, and also the recursion relations characteristic of these functions; 
indeed the operator (18) can be regarded as a product of successive ladder operators for Bessel functions [10] page 60.

To resume, we factorize the translated delta function into the product of a spherical shell function and an angular selector,

$$
\delta\left(r_{1}+r_{2}\right)=4 \pi \sigma\left(r_{1}, r_{2}\right)\left\langle\Omega_{1} \mid-\Omega_{2}\right\rangle
$$

where

$$
\sigma\left(r_{1}, r_{2}\right)=\delta\left(r_{1}-r_{2}\right) / 4 \pi r_{1} r_{2}
$$

and

$$
\left\langle\Omega_{1} \mid-\Omega_{2}\right\rangle=\sum_{l} V(2 l+1)\left\langle\Omega_{1} \Omega_{2} \mid l l ; 00\right\rangle,
$$

the latter expanding as another expression of the closure (12). It follows that the matrix element which is required is

$$
\begin{aligned}
\left\langle l_{1} l_{2} ; \operatorname{lm} \mid \nabla_{1}^{2 n} Y_{l m}\left(\nabla_{1}\right) \delta\left(\mathbf{r}_{1}+\mathbf{r}_{2}\right)\right\rangle & \\
= & v\left(4 \pi\left(2 l_{2}+1\right)\right)\left\langle l_{1} 0 \mid l 0, l_{2} 0\right\rangle D_{n l l_{1} l_{2}}\left(r_{1}\right) \sigma\left(r_{1}, r_{2}\right),
\end{aligned}
$$

for which equations (22), (24), (17) and (15) have been used in turn.

To equate equations (16) and (25) is to represent the non-classical Weber Schafheitlin integral as a generalized function. Thus,

$$
\begin{aligned}
(-1)^{n+l}+ & \frac{1}{4}\left\{\frac{4 \pi(2 l+1)}{\left(2 l_{1}+1\right)\left(2 l_{2}+1\right)}\right\}^{1 / 2}\left\langle l_{1} l_{2} ; \operatorname{lm} \mid \nabla_{1}^{2 n} Y_{l m}\left(\nabla_{1}\right) \delta\left(\mathbf{r}_{1}+\mathbf{r}_{2}\right)\right\rangle \\
& +\left\langle l_{1} 0, l_{2} 0 \mid l 0\right\rangle \\
= & \int d k(2 \pi k)^{2 n+l+2} j_{l_{1}}\left(2 \pi r_{1} k\right) j_{l_{2}}\left(2 \pi r_{2} k\right) \\
= & \pi(-1)^{n+a} D_{n l_{1} l_{2}}\left(r_{1}\right) \sigma\left(r_{1}, r_{2}\right),
\end{aligned}
$$

provided the coupling coefficient $\left\langle l_{1} 0, l_{2} 0 \mid l 0\right\rangle$ does not vanish. In a simple special case, $n=l=a=b=0, l_{1}=l_{2}=c$, the derivatives drop out and equation (26) reveals the orthogonality [20],

$$
\int d k 4 \pi k^{2} j_{c}\left(2 \pi r_{1} k\right) j_{c}\left(2 \pi r_{2} k\right)=\sigma\left(r_{1}, r_{2}\right),
$$

which is plainly a form of the Hankel inversion theorem [18] page 52 and [21] pages 453-464. A further check is provided by considering the limit of $r_{2} \rightarrow 0$ : the second Bessel function vanishes unless $l_{2}=0$, so we may take $l_{1}=l=a$, $c=0$; on the right-hand side $\sigma\left(r_{1}, r_{2}\right) \rightarrow \delta\left(r_{1}\right)$. Equation (26) then reduces to the result of the previous section.

It is no restriction to impose a condition upon $n$ or $l$; and indeed if the exponent of $k$ is small enough we may set $n=0$ and employ the form (18) of the 
differential operator, so that equation (26) appears as

$$
\begin{aligned}
& \int d k(2 \pi k)^{l_{1}+l_{2}-2 c+2} j_{l_{1}}\left(2 \pi r_{1} k\right) j_{l_{2}}\left(2 \pi r_{2} k\right) \\
& =\pi(-1)^{l_{1}+l_{2}} D_{l_{1}-c, l_{1}}\left(r_{1}\right) D_{l_{2}-c, l_{2}}\left(r_{2}\right) \sigma\left(r_{1}, r_{2}\right), \quad\left(0<c \leqslant l_{1}, l_{2}\right),
\end{aligned}
$$

while if the exponent is large enough we may set $c=0$ in equation (21) and employ the form (19), which gives

$$
\begin{aligned}
& \int d k(2 \pi k)^{l_{1}+l_{2}+2 n+2} j_{l_{1}}\left(2 \pi r_{1} k\right) j_{l_{2}}\left(2 \pi r_{2} k\right) \\
& =\pi(-1)^{l_{1}+l_{2}} D_{l_{1} l_{1}}\left(r_{1}\right) D_{10}^{n}\left(r_{1}\right) D_{l_{2} l_{2}}\left(r_{2}\right) D_{10}^{n}\left(r_{2}\right) \sigma\left(r_{1}, r_{2}\right) .
\end{aligned}
$$

Here symmetry appears explicitly when it is recognized that $D^{\dagger}\left(r_{1}\right) \sigma\left(r_{1}, r_{2}\right)=$ $D\left(r_{2}\right) \sigma\left(r_{1}, r_{2}\right)$.

\section{Three Bessel functions}

It is easy to see from the argument of the previous section that an integral over three Bessel functions can be derived by a second translation of the point multipole. The full algebra soon becomes unwieldy, however, so attention will be confined to the simple case of the undifferentiated delta function, by way of illustration. One defines the triple coupled basis [3] page 40 ,

$$
\begin{aligned}
& \left\langle\Omega_{1} \Omega_{2} \Omega_{3} \mid l_{1} l_{2} l_{3} ; l_{12} l m\right\rangle \\
& =\sum_{m_{1} m_{2} m_{3}}\left\langle\Omega_{1} \mid l_{1} m_{1}\right\rangle\left\langle\Omega_{2} \mid l_{2} m_{2}\right\rangle\left\langle\Omega_{3} \mid l_{3} m_{3}\right\rangle\left\langle l_{1} m_{1}, l_{2} m_{2}, l_{3} m_{3} \mid l_{12} l m\right\rangle,
\end{aligned}
$$

which satisfies a closure relation analogous to equation (13). The previous Fourier-transform argument is now repeated until, instead of equation (16), we reach

$$
\begin{aligned}
\left\langle l_{1} l_{2} l_{3} ; l_{12} l m \mid \delta\left(\mathbf{r}_{1}+\mathbf{r}_{2}+\mathbf{r}_{3}\right)\right\rangle \\
=\left\langle l_{12} l m \mid l_{3} 00\right\rangle\left\{\frac{\left(2 l_{1}+1\right)\left(2 l_{2}+1\right)\left(2 l_{3}+1\right)}{4 \pi}\right\}^{1 / 2}\left\langle 00 \mid l_{1} 0, l_{2} 0, l_{3} 0\right\rangle \\
\quad \times(4 \pi)^{3} i^{l_{1}+l_{2}+l_{3} \int d k \cdot k^{2} j_{l_{1}}\left(2 \pi r_{1} k\right) j_{l_{2}}\left(2 \pi r_{2} k\right) j_{l_{3}}\left(2 \pi r_{3} k\right) .}
\end{aligned}
$$

In equation (31) we have a classically respectable integral which has already been treated, and in a similar manner, by Jackson and Maximon [5].

Direct angular integration is another means of separating the angular and radial variables of the twice-translated delta function. If $\left(r_{1}+r_{2}+r_{3}\right)$ is to vanish, we observe that there must be a rotation $R$ with Euler angles $\rho$ that brings the vector triad $\left(\mathbf{r}_{1}, \mathbf{r}_{2}, \mathbf{r}_{3}\right)$ into congruence with any given triangle of sides 
$r_{1}, r_{2}, r_{3} ;$ thus, we can write

$$
\delta\left(\mathbf{r}_{1}+\mathbf{r}_{2}+\mathbf{r}_{3}\right)=8 \pi \tau\left(r_{1}, r_{2}, r_{3}\right) \int d^{3} \rho\left\langle\Omega_{1} \Omega_{2} \Omega_{3}|R(\rho)| \Omega_{1}^{0} \Omega_{2}^{0} \Omega_{3}^{0}\right\rangle,
$$

where relative angles among the coplanar $\Omega_{1}^{0}, \Omega_{2}^{0}, \Omega_{3}^{0}$ are determined by the given triangle. The radial factor $\tau$ can be defined by an angular integral,

$$
\tau\left(r_{1}, r_{2}, r_{3}\right)=(4 \pi)^{-3 / 2}\left\langle 000 ; 0 \mid \delta\left(\mathbf{r}_{1}+\mathbf{r}_{2}+\mathbf{r}_{3}\right)\right\rangle,
$$

which shows that it vanishes unless the lengths $r_{1}, r_{2}, r_{3}$ are capable of forming a closed triangle, and it is evaluated [11] equation 22 from the normalization,

$$
1=\int d^{3} \mathbf{r}_{3} \delta\left(\mathbf{r}_{1}+\mathbf{r}_{2}+\mathbf{r}_{3}\right)=8 \pi r_{1} r_{2} r_{3}^{0} \tau\left(r_{1}, r_{2}, r_{3}^{0}\right)
$$

where $r_{3}^{0} \equiv\left|\mathbf{r}_{1}+\mathbf{r}_{2}\right|$. Now, the scalar product of the spherically-symmetric delta function (32) with the coupled basis (30) is zero unless $l=0$, when the integration over $\Omega_{1}, \Omega_{2}, \Omega_{3}$ is invariant under the rotation $R$, and the integral over $\rho$ is elementary, so that

$$
\left\langle l_{1} l_{2} l_{3} ; 0 \mid \delta\left(\mathbf{r}_{1}+\mathbf{r}_{2}+\mathbf{r}_{3}\right)\right\rangle=(4 \pi)^{3} \tau\left(r_{1}, r_{2}, r_{3}\right)\left\langle l_{1} l_{2} l_{3} ; 0 \mid \Omega_{1}^{0} \Omega_{2}^{0} \Omega_{3}^{0}\right\rangle .
$$

Equating equations (31) and (35), we find

$$
\begin{aligned}
\int d k k^{2} j_{1}\left(2 \pi r_{1} k\right) j_{l_{2}}\left(2 \pi r_{2} k\right) j_{l_{3}}\left(2 \pi r_{3} k\right) \\
=\tau\left(r_{1}, r_{2}, r_{3}\right)\left\langle l_{1} l_{2} l_{3} ; 0 \mid \Omega_{1}^{0} \Omega_{2}^{0} \Omega_{3}^{0}\right\rangle \\
\quad \div\left\{i^{l_{1}+l_{2}+l_{3}}\left[\frac{\left(2 l_{1}+1\right)\left(2 l_{2}+1\right)\left(2 l_{3}+1\right)}{4 \pi}\right]^{1 / 2}\left(\begin{array}{c}
l_{1} l_{2} l_{3} \\
000
\end{array}\right)\right\},
\end{aligned}
$$

provided the coupling coefficient does not vanish. Equation (36) is equivalent to the result of Jackson and Maximon [5].

The effect here of admitting derivatives of the delta function would be to raise the exponent of $k$ in the integrand of equation (36), and to bring in radial derivatives of the right-hand side. A similar result would follow if equation (36) were subjected to the differential operators (20). Such derivatives introduce delta functions at the discontinuities of the function $\tau\left(r_{1}, r_{2}, r_{3}\right)$.

\section{Translating a spherical harmonic}

In any theoretical study of the mechanics of atomic interaction, it is a recurring task to evaluate 'two-centre' integrals which require the displacement of an integrand from one centre to another [11] equation 22, [12], [14] and [17]. It will be shown that the integral of the previous section arises here in a natural way. 
A direct method of translating a generalized function is to integrate over a two-fold translation of the delta function, thus,

$$
f\left(\mathbf{r}_{1}+\mathbf{r}_{2}\right)=\int d^{3} \mathbf{r}_{3} \delta\left(\mathbf{r}_{1}+\mathbf{r}_{2}+\mathbf{r}_{3}\right) f\left(-\mathbf{r}_{3}\right)
$$

which has equation (10) as its Fourier representation. The function to be translated is typically a spherical harmonic of the form, $f(r)=g(r) Y_{L M}(\Omega)$. In the Laplace expansion (13) of this form, some of the terms vanish by the Wigner Eckart theorem, leaving a series with two indices, $l_{1}, l_{2}$. The required scalar product is the integral,

$$
\begin{aligned}
\left\langle l_{1} l_{2} ; L M \mid f\left(\mathbf{r}_{1}+\mathbf{r}_{2}\right)\right\rangle & =\frac{1}{V(2 L+1)} \int r_{3}^{2} d r_{3} g\left(r_{3}\right)\left\langle l_{1} l_{2} L ; 0 \mid \delta\left(\mathbf{r}_{1}+\mathbf{r}_{2}+\mathbf{r}_{3}\right)\right\rangle \\
& =\frac{8 \pi^{2}}{\sqrt{ }(2 L+1)} \int_{\left|r_{1}-r_{2}\right|}^{r_{1}+r_{2}} d r_{3} g\left(r_{3}\right) \frac{r_{3}}{r_{1} r_{2}}\left\langle l_{1} l_{2} L ; 0 \mid \Omega_{1}^{0} \Omega_{2}^{0} \Omega_{3}^{0}\right\rangle,
\end{aligned}
$$

using equations (37), (33)-(35). The rotational invariant that appears in the integrand reduces to a function of two angles of the triangle [5],

$$
\left\langle l_{1} l_{2} L ; 0 \mid \Omega_{1}^{0} \Omega_{2}^{0} \Omega_{3}^{0}\right\rangle=\frac{1}{\sqrt{ }(4 \pi)}\left\langle l_{1} l_{2} ; L 0 \mid-\Omega_{13}^{0},-\Omega_{23}^{0}\right\rangle,
$$

where $\phi_{23}^{0}=\phi_{13}^{0}+\pi$. This is a bipolar harmonic which is simply evaluated according to equation (11) as a finite sum of products of spherical harmonics, the angles of which are determined by the sides of the triangle, as follows:

and

$$
\left.\begin{array}{l}
-\cos \theta_{13}^{0}=\left(r_{1}^{2}-r_{2}^{2}+r_{3}^{2}\right) / 2 r_{1} r_{3}, \\
-\cos \theta_{23}^{0}=\left(r_{2}^{2}-r_{1}^{2}+r_{3}^{2}\right) / 2 r_{2} r_{3} .
\end{array}\right\}
$$

It is also possible to describe the rotational invariant by zonal harmonics of all three angles of the triangle [5].

The above method of translating a spherical harmonic function has an advantage over existing techniques [6] because it recognizes the rotational invariant and preserves the symmetry between the radii $r_{1}$ and $r_{2}$.

\section{References}

[1] B. F. Bayman, “A generalization of the spherical harmonic gradient formula", J. Math. Phys. 19 (1978), 2558-2562.

[2] D. M. Brink and G. R. Satchler, Angular momentum (Clarendon Press, Oxford, 2nd edition, 1968).

[3] U. Fano and G. Racah, Irreducible tensorial sets (Academic Press, 1959).

[4] E. W. Hobson, The theony of spherical and ellipsoidal harmonics (Cambridge University Press, 1931). 
[5] A. D. Jackson and L. C. Maximon, "Integrals of products of Bessel functions", SIAM J. Math. Anal. 3 (1972), 446-460.

[6] A. N. Jette, "A convenient method for evaluating two centre integrals", Internat. J. Quantum Chem. 7 (1973), 131-132, 1040.

[7] D. S. Jones, Generalised functions (McGraw-Hill, 1966).

[8] K. G. Kay, H. D. Todd and H. J. Silverstone, "Dirac delta functions in the Laplace-type expansion of $r^{n} Y_{I}^{m}(\theta, \phi) ", J$. Chem. Phys. 51 (1969), 2359-2362.

[9] A. Messiah, Quantum mechanics (North Holland, 1961).

[10] W. Miller, Lie theory and special functions (Academic Press, 1968).

[11] M. B. Milleur, M. Twerdochlib and J. O. Hirschfelder, "Bipolar angle averages and two-centre, two-particle integrals involving $r_{12}$ ", J. Chem. Phys, 45 (1966), 13-20.

[12] F. P. Prosser and C. H. Blanchard, "On the evaluation of two-centre integrals", J. Chem. Phys. 36 (1962), $1112 ; 43$ (1965), 1086.

[13] E. G. P. Rowe, "Spherical delta functions and multipole expansions", J. Math. Phys. 19 (1978), 1962-1968.

[14] K. Ruedenberg, "Bipolare Entwicklungen, Fourier-transformation und molekulare Mehrzentren-Integrale", Theoret. Chim. Acta 7 (1967), 359-366.

[15] R. A. Sack, "Generating functions for spherical harmonics: part I, three-dimensional harmonics", SIAM J. Math. Anal. 5 (1974), 774-796.

[16] F. D. Santos, "Finite range approximations in direct transfer reactions", Nucl. Phys. A212 (1973), 341-364.

[17] H. J. Silverstone, "Expansion about an arbitrary point of three-dimensional functions involving spherical harmonics by the Fourier-transform convolution theorem", J. Chem. Phys. 47 (1967), 537-540.

[18] I. N. Sneddon, Fourier transforms (McGraw-Hill, 1951).

[19] J. A. Stratton, Electromagnetic theory (McGraw-Hill, 1941).

[20] Y. Suzuki, "Some formulae on Bessel and Legendre functions", J. Coll. Arts Sci. Chiba Univ. 3 (1962), 441-446.

[21] G. N. Watson, A treatise on the theory of Bessel functions (Cambridge University Press, 2nd edition 1944).

\section{C.S.I.R.O. Division of Chemical Physics \\ P. O. Box 160}

Clayton

Victoria 3168 